https://doi.org/10.37208/tgn27326

\section{Expansion in recorded range of fossorial water voles in Glasgow, Scotland}

\author{
C.J. McInerny \\ School of Life Sciences, University of Glasgow, \\ Glasgow G12 8QQ \\ E-mail: chris.mcinerny@ glasgow.ac.uk
}

The water vole (Arvicola amphibius) is a rare mammal throughout Scotland and the U.K. with numbers suffering dramatic declines due to changes in agricultural practices and through predation by introduced American minks (Neovison vison) (Rushton et al., 2000; Aars et al., 2001; Telfer et al., 2003; Strachan, 2004; Dean et al., 2016). Thus the colonies in east Glasgow, where large numbers are found, are of national importance, and are unusual in being present in urban grasslands far from water with the voles having a fossorial lifestyle, digging holes and living underground (Stewart et al., 2017; McInerny, 2018; Stewart et al., 2019).

I have been studying the populations of water voles in east Glasgow for a number of years, surveying their distribution during 2018 and 2019. Animals were detected by visual surveys during March, April and May when they were more visible. At this time of the year voles, having been mostly underground through the winter, spend much time feeding on grass near their holes above ground, and are particularly visible due to the short and flattened sward (Fig. 1). Their presence during the surveys was also established by the detection of holes, diggings, pellets and clipped grass, indicative of the species (Stewart et al., 2019). Water voles engage in much digging and excavation in early spring and leave half-consumed grass, making their detection straightforward (Fig. 2).

The surveys over two springs during 2018 and 2019 revealed the presence of fossorial water voles across many parts of east Glasgow (Fig. 3), with animals and diggings located at numerous locations from Bishop Loch (NS687664) in the east to Hogganfield Loch (NS646676) in the west. All were far from water, with many located in public parks, but with others on road verges (Fig. 1B) and neglected ground. This distribution was similar to that noted by others (Stewart et al., 2017; Wijas et al., 2019), although in many areas higher densities were detected and new sites were found. A common feature of the locations of animals and burrows was the absence of management of the ground, with grass always uncut (Stewart et al., 2019). Many such unmanaged areas are found throughout east Glasgow (Fig. 2). Because most of these were monitored for the presence of water voles during the surveys of 2018 and 2019, I believe that the distribution shown in Fig. 3 gives a reasonably accurate impression of their range in east Glasgow during these two years.

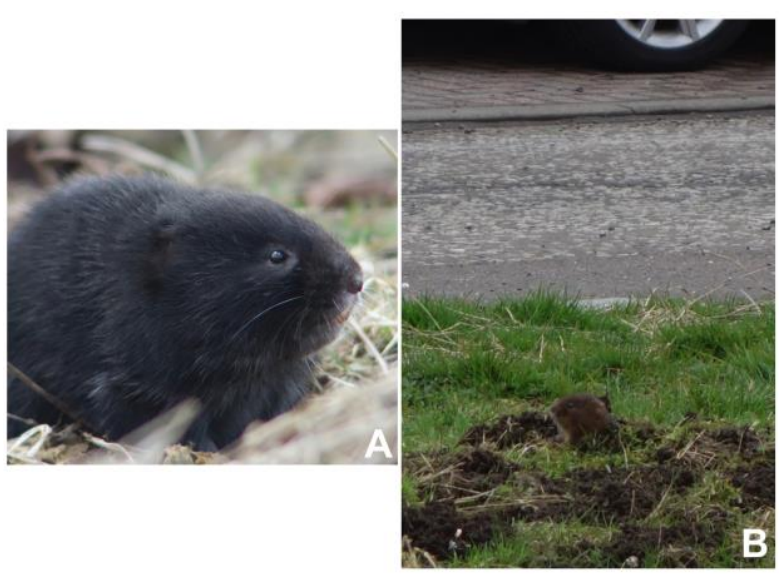

Fig. 1. Water voles (Arvicola amphibius), Glasgow, Scotland. (A) Black animal, Ruchazie, Glasgow, 23rd March 2018. (B) Brown animal, Easterhouse, 18th April 2019. (Photos: Chris McInerny)

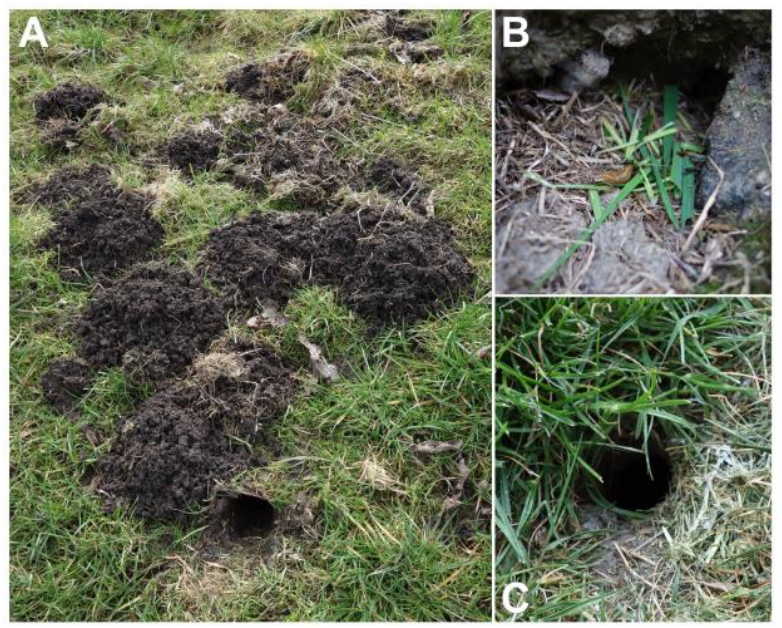

Fig. 2. Evidence of the presence of water voles (Arvicola amphibius), Glasgow, Scotland. (A) Burrow entrance and diggings, Cranhill Park, 23rd March 2018. (B,C) Clipped grass at burrow entrances, Ruchill Park, Glasgow, 15th May 2020. Burrow entrances $c a .5 \mathrm{~cm}$ in diameter. (Photos: Chris McInerny)

Through the spring of 2020 travel restrictions due to the COVID-19 pandemic prevented me from visiting east Glasgow. I was, however, able to monitor areas in the West End of Glasgow near my home during "lockdown" walks. The preferred habitat of water voles, uncut grass verges and neglected land, was surveyed within $3 \mathrm{~km}$ of Glasgow Botanic Gardens. Most areas revealed no evidence of animals, but burrows and fresh clipped grass were discovered in Ruchill Park, with at least eight burrows located at different parts of the park (Fig. 2). Ruchill Park is about $7 \mathrm{~km}$ west of the nearest fossorial water voles in east Glasgow at Hogganfield Loch (Fig. 4). This is the first time that fossorial water 


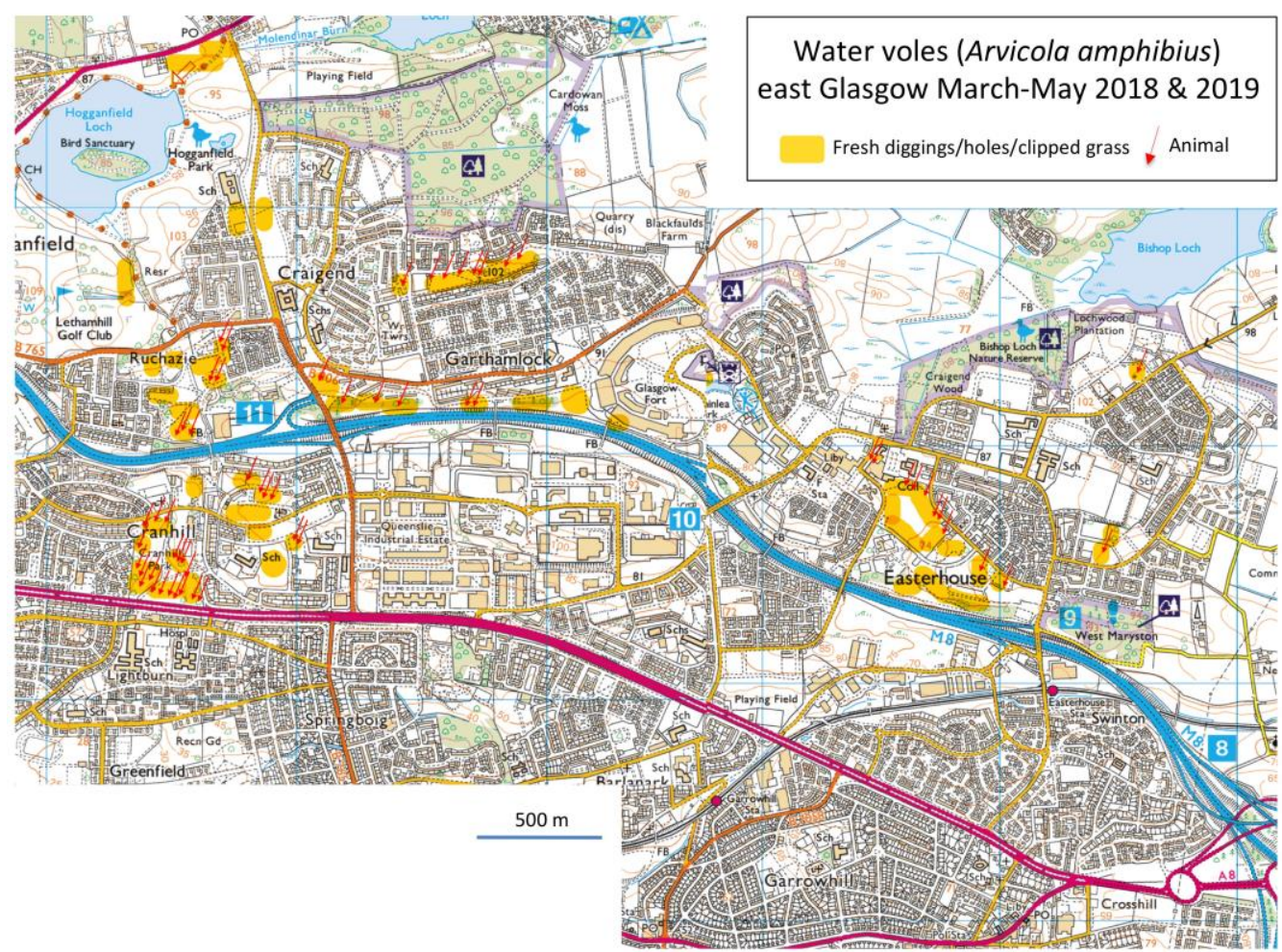

Fig. 3. Water vole (Arvicola amphibius) distribution in east Glasgow, Scotland, March-May, 2018 and 2019.

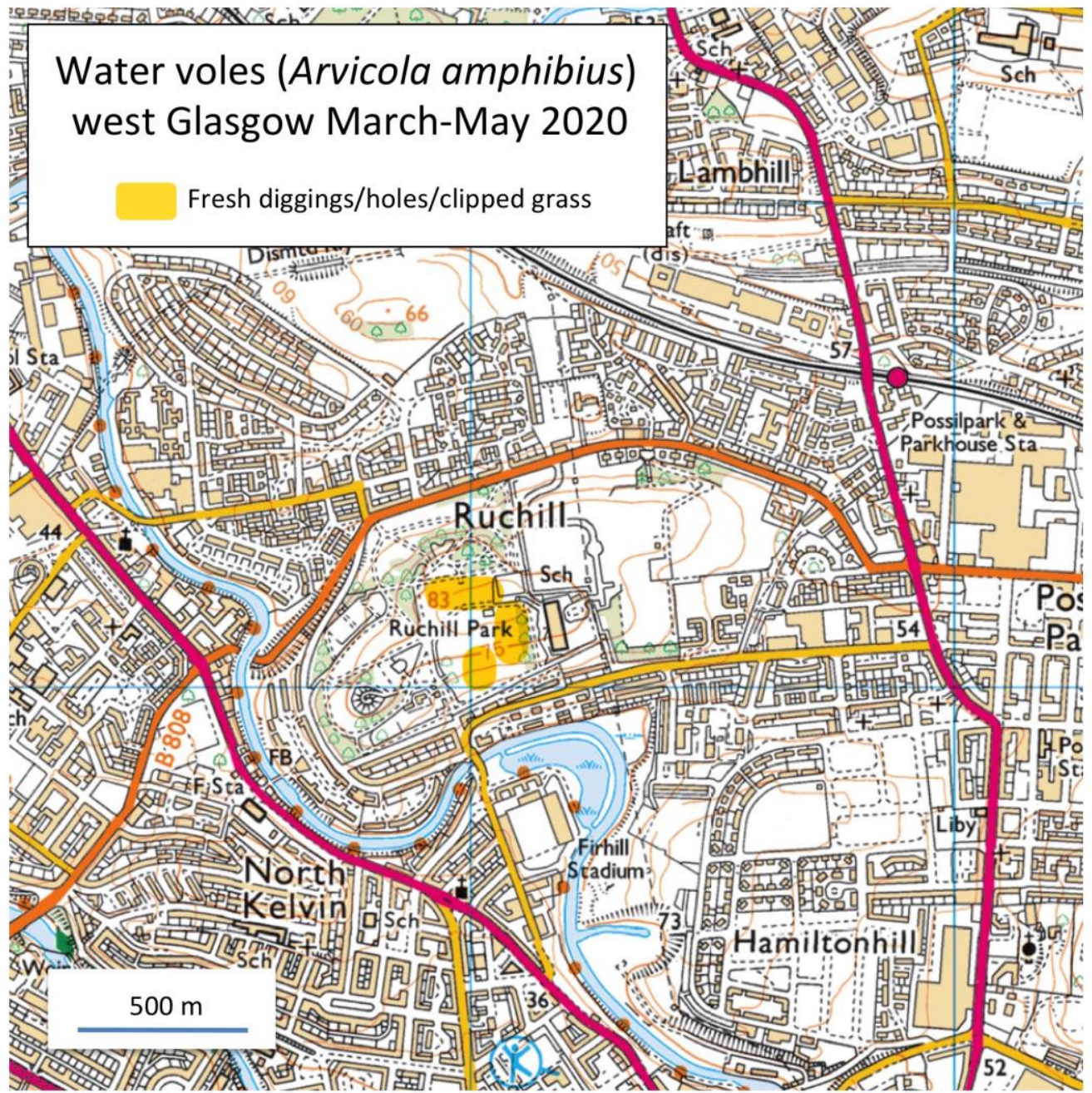

Fig. 4. Water vole (Arvicola amphibius) distribution in west Glasgow, Scotland, March-May, 2020. 
voles have been found in the West End of the city, although animals have occasionally been observed elsewhere in west Glasgow on watercourses (Wijas et al., 2019). These are likely to be the aquatic rather than the fossorial form.

I have subsequently searched for evidence of fossorial water voles between Ruchill Park and Hogganfield Loch, but with no success. It is therefore unclear how they colonised Ruchill, although being fossorial they may be derived from the large east Glasgow populations. The species distribution has been noted to be patchy elsewhere (Lawton \& Woodroffe, 1991), with this being due in some areas to predation by American mink and habitat fragmentation (Rushton et al., 2000). However, these seem unlikely reasons to explain the distribution in Glasgow as much apparently suitable habitat is present throughout the city and American minks are rare to largely absent (Wijas et al., 2019; pers. obs.). Predation of fossorial water voles by red foxes (Vulpes vulpes) has been reported (Weber \& Aubry, 1993). Since Glasgow has a rich population of red foxes, it is possible that this predator may influence the urban water vole distribution. Furthermore, birds of prey such as common buzzard (Buteo buteo), common kestrel (Falco tinnunculus) and peregrine (Falco peregrinus) are all present in the city and have been observed hunting voles (McInerny, 2018), so may play a role.

Finally, it is important to note that a number of sites in east Glasgow where water voles were detected during the 2018 and 2019 surveys are being developed for housing. Trapping of voles was observed and these are presumably moved to other protected sites. Even so, it appears that much habitat occupied by water voles will be lost, which, considering the species' rarity and status in the U.K., is unfortunate, especially when the unusual fossorial behaviour of the Glasgow population is considered.

\section{REFERENCES}

Aars, J., Lambin, X., Denny, R., \& Griffin, A. (2001). Water vole in the Scottish uplands: distribution patterns of disturbed and pristine populations ahead and behind the American mink invasion front. Animal Conservation 4, 187-194. https://doi.org/10.1017/S1367943001001226

Dean, M., Strachan, R., Gow, D. \& Andrews, R. (2016). The Water Vole Mitigation Handbook. The Mammal Society, London.

Lawton, J.H., \& Woodroffe, G.L. (1991). Habitat and the distribution of water voles: why are there gaps in a species' range? Journal of Animal Ecology 60, 7991. https://doi.org/10.2307/5446

McInerny, C.J. (2018). Peregrine hunting water voles in Glasgow. Scottish Birds 38, 240-241.

Rushton, S.P., Barreto, G.W., Cormack, R.M., MacDonald, D.W. \& Fuller, R. (2000). Modelling the effects of mink and habitat fragmentation on the water vole. Journal of Applied Ecology 37, 475-490. https://doi.org/10.1046/j.1365-2664.2000.00504.x

Stewart, R.A., Clark, T.J., Shelton, J., Stringfellow, M.,
Scott, C., White, S.A. \& McCafferty, D.J. (2017). Urban grasslands support threatened water voles. Journal of Urban Ecology 3, 1-17. https://doi.org/10.1093/jue/jux007

Stewart, R.A., Jarrett, C., Scott, C., White, S.A. \& McCafferty, D.J. (2019). Water vole (Arvicola amphibius) abundance in grassland habitats of Glasgow. The Glasgow Naturalist 27(1), 10-19. https://doi.org/10.37208/tgn27102

Strachan, R. (2004). Conserving water voles: Britain's fastest declining mammal. Water and Environment Journal 18, 1-4. https://doi.org/10.1111/j.1747-6593.2004.tb00483.x

Telfer, S., Dallas, D.F., Aars, J., Piertney, S. B., Stewart, W.A. \& Lambin, X. (2003). Demographic and genetic structure of water voles (Arvicola terrestris) on Scottish islands. Journal of Zoology 259, 23-29. https://doi.org/10.1017/S0952836902003321

Weber, J.M. \& Aubry, S. (1993). Predation by foxes, Vulpes vulpes, on the fossorial form of the water vole, Arvicola terrestris scherman, in western Switzerland. Journal of Zoology 229, 553-559. https://doi.org/10.1111/j.1469-7998.1993.tb02656.x

Wijas, B.J., Stewart, R.A. \& McCafferty, D.J. (2019). Potential risk of American mink to water vole populations in east Glasgow. The Glasgow Naturalist 27(1), 84-86.

https://doi.org/10.37208/tgn27122 